\title{
AVALIAÇÃO DE ADJUVANTES EM NOVAS FORMULAÇÕES DE VACINA TRÍPLICE
}

BACTERIANA

ANA FABÍOLA ROLLO DE OLIVEIRA PRESTES

Dissertação apresentada ao Programa de PósGraduação Interunidades em Biotecnologia USP/Instituto Butantan/IPT, para obtenção do Título de Mestre em Biotecnologia.

Área de concentração: Biotecnologia

Orientadora: Dra. Waldely de Oliveira Dias 


\section{RESUMO}

Prestes AFRO. Avaliação de adjuvantes em novas formulações de vacina tríplice bacteriana [Dissertação]. São Paulo: Instituto Butantan, Universidade de São Paulo; 2009.

A vacina tríplice bacteriana (DTP) é constituída de toxóides diftérico (DT) e tetânico (TT), e suspensão bacteriana destoxificada de Bordetella pertussis (P). A imunização com a DTP provou-se bastante eficaz no controle da coqueluche, mas foi associada a efeitos colaterais, levando ao desenvolvimento da vacina pertussis acelular, menos tóxica, composta por antígenos bacterianos purificados. Devido a seu alto custo, o Instituto Butantan desenvolveu duas novas propostas de vacina pertussis: acelular $(\mathrm{Pa}$ - utilizando filtrado de cultivo destoxificado da bactéria) e celular low ( $\mathrm{P}_{\text {low }}$ - com baixo teor de lipopolissacarídeo - LPS), obtidas a partir da linha de produção da vacina pertussis celular, sem encarecer o processo. O objetivo deste projeto foi o estudo imunomodulatório em modelo animal, das vacinas tríplices bacterianas (DTP, DTPa e DTP $_{\text {low }}$ ) adicionadas de diferentes adjuvantes: vitamina A, monofosforil lipídeo A (MPL) de $B$. pertussis, surfactante pulmonar de origem suína e BCG, comparativamente ao tradicional hidróxido de alumínio. Os resultados obtidos mostraram que a troca dos componentes pertussis na vacina tríplice não interfere no comportamento dos toxóides na indução de anticorpos séricos, induzindo altos títulos de IgG contra os antígenos vacinais, sem diferença significativa entre os adjuvantes. As vacinas induziram níveis equilibrados de $\operatorname{IgG} 1 / \operatorname{IgG} 2$ a anti-pertussis e a $\mathrm{DTP}_{\text {low }}$ mostrou-se menos reatogênica, induzindo níveis significativamente menores de IL-6 sérica. A adição de MPL sugeriu tendência de proteção contra a colonização nasotraqueal no grupo imunizado com DTPa e levou à indução de IFN- $\gamma$ nos grupos imunizados com DTP e DTPa, sugerindo possível atividade imunomodulatória para Th1. Esperamos que o projeto forneça subsídios para a utilização de uma formulação menos reatogênica para a vacina tríplice bacteriana, em escala industrial, e uma possível substituição do hidróxido de alumínio, que sabidamente contribui para aumentar as reações adversas da vacina, principalmente em crianças, nas primeiras doses da escala vacinal.

Palavras-chaves: Coqueluche. Vacinas. Lipopolissacarídeo. Vitamina A. Adjuvantes imunológicos. Alumínio. 


\begin{abstract}
Prestes AFRO. Evaluation of adjuvants in new triple bacterial vaccine formulation [Master thesis]. São Paulo: Instituto Butantan, Universidade de São Paulo; 2009.

The triple bacterial vaccine (DTP) is composed of diphtheria (DT) and tetanus toxoids (TT), and suspension of inactivated Bordetella pertussis cells (P). The immunization with DTP showed high effectiveness in the control of whooping-cough, but was associated with adverse effects, leading up to the development of an acellular pertussis vaccine, less toxic and formulated with purified bacterial antigens. Due to its expensive price, the Butantan Institute developed two new pertussis vaccines proposals: acellular ( $\mathrm{Pa}$ - utilizing filtered bacterial detoxified culture) and low cellular $\left(\mathrm{P}_{\text {low }}-\right.$ less lipopolysaccharide), obtained from the same production line of cellular pertussis vaccine, without raising the prices of the process. The objective of this project was the immunomodulatory study of the triple bacterial vaccines (DTP, DTPa and DTP low $_{\text {lo }}$ in animal model, with different adjuvants: vitamin A, monophosphoryl lipid A (MPL) of B. pertussis, porcine pulmonary surfactant and BCG vaccine, comparatively with the traditional aluminum hydroxide. The results showed that the modification of the pertussis components in the triple bacterial vaccine does not intervene in the antibody induction of the toxoids, inducing high IgG titers against vaccines antigens, without significant differences between the adjuvants. The vaccines induced balanced levels of $\operatorname{IgG} 1 / \operatorname{IgG} 2 \mathrm{a}$ anti-pertussis and $\mathrm{DTP}_{\text {low }}$ showed to be less rectogenic, inducing lower levels of serum IL-6. The use of MPL suggested to induce higher protection against nasotracheal colonization in DTPa group and induced IFN- $\gamma$ in the DTP and DTPa groups, suggesting a possible immunemodulatory activity for Th1. We hope this project offer subsidies to the utilization of a less reactogenic formulation to triple bacterial vaccine, in industrial scale, and a possible substitution of the aluminum hydroxide, what is known to contribute to the adverse reactions of the vaccine, mainly in children, in the first vaccine doses.
\end{abstract}

Key words: Whooping cough. Vaccines. Lipopolysaccharide. Vitamin A. Immunological adjuvants. Aluminum. 


\section{INTRODUÇÃO}

As vacinas são consideradas uma das maiores intervenções médicas contra doenças infecciosas (Pashine et al., 2005) e sua importância é ilustrada pelo fato de que os programas de vacinação mundiais levaram a uma completa, ou quase completa, erradicação de algumas dessas doenças (Abbas et al., 2003).

O Instituto Butantan possui tradição pioneira na área de saúde pública, realizando

pesquisas de novos imunobiológicos. É responsável pela produção de mais de $80 \%$ das vacinas utilizadas no Brasil, sendo o produtor nacional da vacina tríplice bacteriana (DTP - difteria, tétano e pertussis).

Há quase 20 anos, o Centro de Biotecnologia do Instituto Butantan vem desenvolvendo pesquisa e desenvolvimento para a produção de novas vacinas pertussis, com vistas à obtenção de produtos menos tóxicos e tão eficientes na prevenção da coqueluche quanto a vacina tradicional. Assim, foi desenvolvido um processo inédito de produção de uma vacina pertussis acelular, em processo simples e acoplado ao da produção da vacina pertussis celular tradicional, com preparações semi purificadas do filtrado de cultivo, compostas por uma mistura de antígenos excretados para o meio de cultura (Dias et al., 2002a, Furuyama, 2001). A vacina pertussis acelular do Instituto Butantan vem sendo exaustivamente testada em estudos pré-clínicos e tendose mostrado segura e imunogênica (Dias et al., 1994, 2004a, 2007).

Mais recentemente, foi desenvolvida uma nova vacina pertussis celular (Dias et al., 2004b; Kubrusly et al., 2006), que inclui etapas de destoxificação do componente lipopolissacarídico (LPS) da bactéria, um dos principais responsáveis pela toxicidade residual das vacinas pertussis celulares. Nesse processo, é obtido como subproduto o monofosforil lipídeo A (MPL) (Raw et al., 2006), um componente não tóxico que vem sendo avaliado como adjuvante em formulações vacinais. Esses quatro produtos, a vacina pertussis celular tradicional $(\mathrm{P})$, a vacina pertussis acelular $(\mathrm{Pa})$, a vacina pertussis celular com baixa quantidade de LPS $\left(\mathrm{P}_{\text {low }}\right)$ e o MPL são obtidos na mesma linha de produção, em processos simples e de baixo custo (Figura 1). 


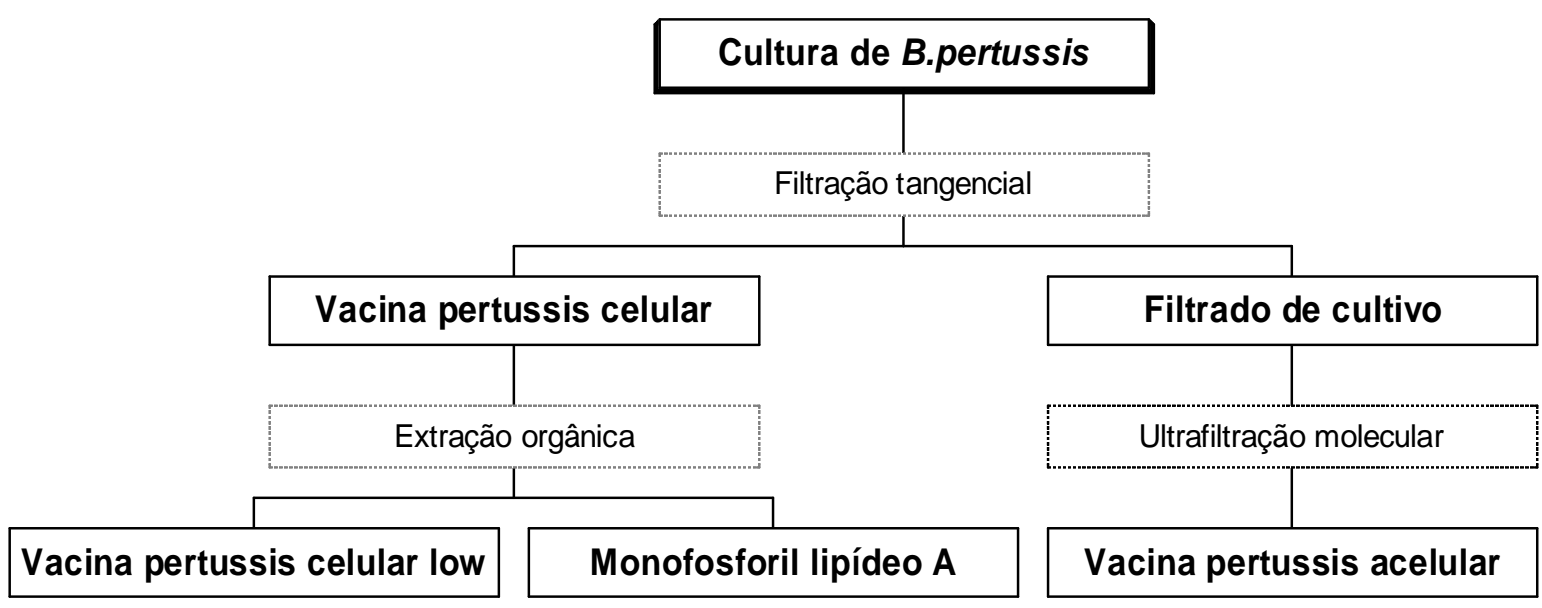

Figura 1 - Esquema da linha de produção de vacinas pertussis e do adjuvante em desenvolvimento no Instituto Butantan. A cultura de B. pertussis passa por uma filtração tangencial, obtendo-se a vacina pertussis celular e o filtrado de cultivo; a partir da vacina celular, por uma extração orgânica, tem-se a vacina pertussis celular low e o monofosforil lipídeo A. A vacina pertussis acelular é obtida através de uma ultrafiltração molecular do filtrado de cultivo.

\subsection{Vacina Tríplice Bacteriana}

A vacina tríplice bacteriana tradicional começou a ser administrada a partir dos anos de 1920 em países industrializados, principalmente para as classes mais ricas da população. Em 1974, foi criado o Programa Internacional de Imunização, e a vacina DTP passou a integrar esse programa, o qual foi adotado por vários países, aumentando a cobertura de imunização contra esses antígenos (Clements e Griffths, 2002).

A vacina tríplice bacteriana convencional é constituída de antígenos protetores contra a difteria, o tétano e a coqueluche. Os toxóides tetânico e diftérico possuem elevado poder imunogênico, principalmente quando associados aos adjuvantes minerais, entre eles o hidróxido de alumínio (Vacinas: Vacina Tríplice Bacteriana [...], 2008).

O desempenho imunogênico da vacina pertussis pode variar de acordo com a constituição vacinal, porém sua eficácia é geralmente elevada, diminuindo a freqüência e a gravidade da coqueluche em crianças e jovens, em proporções consideráveis (Vacinas: Vacina Tríplice Bacteriana [...], 2008). A vacina pertussis pode ser celular, constituída por células bacterianas 
inteiras destoxificadas de $B$. pertussis, podendo provocar reações de sensibilidade, vermelhidão, choro persistente, febre, irritação e raramente convulsão, e reações locais mais intensas quando administrada com sais de alumínio (Clements e Griffths, 2002; Raguckas et al., 2007). A preocupação com essas possíveis reações levou ao desenvolvimento das vacinas pertussis acelulares, constituídas de antígenos bacterianos, obtidos do sobrenadante ou filtrado de cultura da bactéria (Dias et al., 2004a).

As vacinas pertussis de células inteiras estão sendo substituídas gradualmente pelas vacinas pertussis acelulares, menos reatogênicas e igualmente imunogênicas e eficazes. Os componentes que normalmente fazem parte das vacinas pertussis acelulares são o toxóide pertussis (PT), a hemaglutinina filamentosa (FHA), a pertactina (PRN) e fímbrias (FIM) (Denöel, 2005), com variações qualitativas e quantitativas, de acordo com cada produtor.

A vacina DTP com componente pertussis celular é extremamente eficiente, e sua larga aplicação tem conduzido a uma redução drástica na incidência da coqueluche em países industrializados, mas não é recomendada para indivíduos maiores que 7 anos devido ao elevado número de efeitos colaterais associados à vacina celular (Dias et al., 2004a; Leef et al., 2000; Recommendations for whole-cell pertussis vaccine, 2007; Vacinas: Vacina Tríplice Bacteriana [...], 2008). Entretanto, a epidemiologia de pertussis está mudando, e apesar da alta cobertura de vacinação da população, foi observado um aumento significativo na incidência dessa doença em crianças, adolescentes e adultos, em vários países durante as duas últimas décadas (Denöel et al., 2005; Leung et al., 2007). Até $25 \%$ dos adultos com tosse prolongada podem apresentar evidências de infecção recente por Bordetella pertussis, e agem como reservatório de infecção para crianças vulneráveis. Isso antecipou, em alguns países, o licenciamento de vacina tríplice bacteriana com componente pertussis acelular (DTPa) (Barreto et al., 2007; Cherry, 1999; Pachichero e Casey, 2005), que vem se mostrando segura, imunogênica e efetiva quando administrada como reforço em adolescentes e adultos, reduzindo a carga da coqueluche entre receptores vacinais e impedindo a transmissão de $B$. pertussis aos recém nascidos (CDC, 2006; von König et al., 2002). Alguns países, como os Estados Unidos, Canadá, França e a Alemanha, já introduziram a DTPa como reforço para maiores de 10 anos de idade (Barreto et al., 2007; Campins-Martí et al., 2002; Carvalho e Pereira, 2006; Leung, 2007). No Brasil, a vacina DTPa licenciada, até o momento, é constituída por três componentes imunogênicos de $B$. pertussis, PT, PRN e FHA (Carvalho e Pereira, 2006). A vacina pertussis acelular nos moldes similares a de 
outros paises tem custo de produção consideravelmente mais elevado do que a celular, inviabilizando sua distribuição gratuita pelo Sistema Único de Saúde no Brasil. Na tecnologia inédita desenvolvida pelo Instituto Butantan para produção de vacina pertussis acelular prevalece a utilização de etapas simples de processamento, resultando em um produto final de baixo custo.

A vacina DTP $_{\text {low, }}$ com componente pertussis com baixo teor de LPS (Dias et al., 2004b), produzida pelo Instituto Butantan foi avaliada em vários experimentos animais, no próprio Instituto e no Nederlands Vaccin Institut - Holanda (Sakahushi MA, 2008 - em fase de elaboração) ${ }^{1}$, apresentando resultados seguros e menor reatogenicidade quando comparada à vacina pertussis celular tradicional. Em ensaio de campo de Fase I, em recém-nascidos, a DTP ${ }_{\text {low }}$ induziu resposta imune humoral e celular semelhante à DTP, sem efeitos colaterais relevantes (Zorzeto et al., 2007a, 2007b).

\subsection{Adjuvantes}

Adjuvante ("adjuvare" = auxiliar) é o nome aplicado a substâncias usadas juntamente com antígenos, para auxiliar ou potencializar a resposta imunológica (Lima, 2008), em certos casos, também chamados de imunoestimulantes (Vacinas Inativadas: os adjuvantes, 2008). Em 1925, estudos pioneiros de Ramon demonstraram ser possível aumentar a resposta imunológica contra toxinas tetânicas ao adicionar à fórmula substâncias como amido, lecitina ou saponinas (Vacinas Inativadas: os adjuvantes; 2008). Assim, há mais de 80 anos os adjuvantes vêm sendo utilizados em experimentos científicos e na vacinação humana e animal, nos quais seu uso adequado dispensa doses elevadas e aplicações freqüentes do antígeno sensibilizante (Lima, 2008).

Várias substâncias têm sido avaliadas como aditivos em vacinas, na tentativa de aumentar sua eficácia. Apesar do excesso de opções, somente os sais de alumínio têm ampla aceitação como adjuvantes de vacinas para humanos e até mesmo de uso veterinário (Pashine et al., 2005).

Os adjuvantes têm diversos mecanismos de ação e devem ser selecionados baseados na via de administração e na resposta imune desejada (Clements e Griffths, 2002). A seleção do

\footnotetext{
${ }^{1}$ Sakahushi MA. São Paulo, 2008. (em fase de elaboração).
} 
adjuvante não visa somente o aumento da resposta imune, mas sua modulação para o sistema Th1 ou Th2, à medida que outras imunoglobulinas e células mediadoras de imunidade são geradas (Cox e Coulter, 1997). Os adjuvantes incrementam o afluxo de linfócitos e modulam a permanência de outras células inflamatórias no local de deposição do antígeno, potencializando a resposta imunológica do hospedeiro (Vacinas Inativadas: os adjuvantes; 2008). Inicialmente, o mecanismo de ação dos adjuvantes foi explicado pela propriedade de reter os antígenos no sítio de inoculação (é o caso dos adjuvantes oleosos, por exemplo). Mais tarde, foram considerados imunorreguladores do equilíbrio entre a resposta imunológica protetora, a tolerância e a supressão, em mecanismo relacionado com o processo inflamatório inespecífico, acompanhado do recrutamento de fagócitos mononucleares para o local da injeção. O mecanismo mais aceito é o que se baseia na capacidade dos adjuvantes de induzirem de maneira seletiva uma das duas subpopulações de células-T auxiliares: Th1 ou Th2 (Lima, 2008).

Dois importantes fatores para o desenvolvimento de vacinas são: apresentação de antígenos altamente purificados, mais efetivos e em pequena quantidade; e, direcionamento da resposta imune em caminhos específicos. O primeiro passo pode ser resolvido com uma ampla variedade de adjuvantes de vacinas. O segundo requer cuidado na seleção entre eles, para permitir o direcionamento da resposta imunológica da melhor maneira (Lin e Jones, 1997). Por exemplo, a expansão da resposta de células $\mathrm{B}$, resposta de células $\mathrm{T}$ citotóxicas, ou realçando cada uma das respostas Th1 ou Th2.

A natureza química dos adjuvantes utilizados em vacinas é bastante variada. Alguns dos mais utilizados são: a) os sais de alumínio, tais como fosfato e hidróxido de alumínio que são aprovados para uso clínico em humanos e animais (Lima, 2008), induzindo produção de respostas imunológicas 90\% para uma tendência Th2 (Cox e Coulter, 1997); b) uma combinação de sais de alumínio com MPL; c) combinação de MPL com uma fração purificada do extrato de saponina de Quillarja saponina (QS-21) e uma emulsão de óleo em água; d) endotoxinas bacterianas e derivados como LPS que induzem principalmente resposta Th1 (Pashine et al., 2005; Lima, 2008), como o MPL, adjuvante na vacina para alergia Pollinex Quattro, licenciado em alguns países (Brennan e Dougan, 2005; Lima, 2008); e) e óleos minerais e vegetais (Lima, 2008). 


\subsubsection{Sais de Alumínio}

Os géis de alumínio vêm sendo usados como adjuvantes em vacinas há 80 anos, desde que Glenny et al. (1926) descobriram que o toxóide diftérico tinha suas propriedades antigênicas aumentadas com a adição de suspensão de alumínio, tornando-se, assim, adjuvante padrão para a vacina tríplice bacteriana.

Três formas de sais de alumínio são usadas em vacinas: sulfato potássico de alumínio (alúmem de potássio), sulfato de alumínio e hidróxido de alumínio, dos quais o último parece ser o mais imunogênico (Baylor et al., 2002; Jefferson et al., 2004). Vacinas com alumínio têm um longo registro de segurança, apesar de causarem algumas reações locais de gravidade variada, como nódulos, granulomas, eritemas, e uma síndrome progressiva caracterizada por desgaste muscular e grave fadiga chamada miofascite macrofágica (Clements e Griffths, 2002; Jefferson et al., 2004). Sabe-se que as reações locais aumentam com sucessivas doses de DTP, DTPa e dT (vacina dupla bacteriana, difteria e tétano), causando inchaço no membro inteiro em aproximadamente $5 \%$ dos casos, fato que agora está sendo relacionado ao hidróxido de alumínio (Mielcarek et al., 2006; Rennels et al., 2002). Além de não ser eficaz com todos os antígenos, pouco se sabe sobre o mecanismo pelo qual a reposta imune é aumentada. Sugere-se que a ação dos sais de alumínio seja formação de depósito de antígeno e imunoestimulação (HogenEsch, 2002), estimulando principalmente resposta humoral (tipo Th2), associada à produção de IL-4 e $\mathrm{IgG1}$, induzindo reposta celular de forma reduzida devido ao retardo na apresentação de linfócitos T CD4 ${ }^{+}$no sítio inflamatório (Resende et al., 2004).

O uso periódico de vacinas com hidróxido de alumínio pode ter papel importante na patogênese da doença de Alzheimer, na miofascite macrofágica e encefalopatias, e pode estar relacionado com aumento na incidência de doenças alérgicas em indivíduos predispostos, uma vez que aumenta a produção de IgE (Baylor et al., 2002; Goto et al., 1993). Efeitos citotóxicos provocados pelo hidróxido de alumínio foram observados por Goto et al. (1993), como aumento da permeabilidade vascular e aumento da liberação de lactato desidrogenase pelos fagócitos, indicando que este composto pode ser tóxico para os macrófagos. Cristais de alumínio foram observados por Authier et al. (2006) e Gherardi et al. (2001) no citoplasma de macrófagos, evento associado a doses repetidas deste componente no local da injeção. Segundo Authier et al. 
(2006), apenas $10 \mu \mathrm{L}$ de hidróxido de alumínio, injetado por via intramuscular em ratos, foi suficiente para o acúmulo em macrófagos, formando lesões como miofascite macrofágica.

Se o hidróxido de alumínio vier a tornar-se inaceitável como adjuvante, muitas vacinas correntes, formuladas com alumínio, podem deixar de ser produzidas, já que, para sua substituição, outros compostos precisam ser estudados quanto à segurança e eficácia, antes de serem licenciados. Tal fato causaria uma crise global no abastecimento de vacinas, ameaçando os programas de imunização, principalmente em países em desenvolvimento, uma vez que o alumínio é um componente que não onera o custo final da vacina.

\subsubsection{Vitamina A}

Descoberta em 1909, a vitamina A é conhecida também como ácido retinóico ou retinol (vitamina A de origem animal). A vitamina A, um antioxidante obtido dos carotenóides vegetais, está envolvida nas reações da visão e crescimento, e diferenciação de tecidos, sendo essencial para o cabelo e os ossos. Tem também papel importante no desenvolvimento e manutenção de um sistema imune saudável, sendo considerada agente antiinfeccioso (Dias et al., 2002b; Marzzoco e Torres, 1999; Programa nacional de [...], 2005; Vitamin A and your bones, 2005). É uma vitamina lipossolúvel, absorvida juntamente com os lipídeos e transportada pelas lipoproteínas plasmáticas (Marzzoco e Torres, 1999). Aproximadamente 90\% da vitamina A do organismo é armazenada no fígado; o restante é armazenado nos depósitos de gordura, pulmões e rins (Vitamina A, 2008). A deficiência de vitamina A (hipovitaminose A) é uma doença nutricional grave, causa mais freqüente de cegueira prevenível entre crianças, contribuindo para o aumento das mortes e doenças infecciosas na infância, como sarampo e pneumonia (Jason et al., 2002; Programa nacional de [...], 2005). A deficiência de vitamina A está associada com a diminuição da resistência a infecções e aumento da morbidade e mortalidade relacionada com doenças infecciosas com sintomas pulmonares e sistêmicos, principalmente em crianças, interferindo diretamente na resposta imune primária (Hussey e Klein, 1990; Jason et al., 2002; Semba et al., 1994). 
A suplementação de vitamina A em bebês é de 100.000UI, entretanto tem sido recomendada uma dose adicional de 50.000UI junto com a administração das vacinas DTP e pólio (Sommer e Davidson, 2002). A suplementação com retinol durante a imunização aumenta rapidamente a resposta imune (Ross, 1996; Semba et al., 1994). O mecanismo pelo qual isso acontece ainda não é claro. Semba et al. (1994) encontraram altos títulos de IgG1 anti-tétano, nas respostas primárias e secundárias, em crianças suplementadas com vitamina A e imunizadas com a vacina de tétano. A co-administração de vitamina A com a vacinação de sarampo e DTP elevou significativamente a resposta de anticorpos induzidos pela vacina em bebês, sugerindo seu potencial beneficio no início da vida (Ma e Ross, 2005).

Estudos têm mostrado que retinóides têm efeito adjuvante quando administrados na imunização. Segundo Dias et al. (2002b) a vitamina A teve efeito adjuvante na resposta humoral de camundongos imunizados com a vacina DTP, mostrando um indiscutível efeito de reforço e níveis similares ou mais altos de anticorpos, quando comparados aos induzidos por esses antígenos, com hidróxido de alumínio. Além disso, a vitamina A usada como adjuvante em vacinas pode também auxiliar na hipovitaminose $\mathrm{A}$ em crianças, o que interfere diretamente na resposta imune primária.

\subsubsection{Surfactante Pulmonar}

Embora a função primária do surfactante seja a de reduzir a tensão superficial nos alvéolos, alguns estudos indicam que o surfactante pulmonar também pode influenciar a função imune, com atividade na defesa inata do hospedeiro (Crouch e Wright, 2001; Mizuno, 2006). Proteínas do surfactante interagem com ampla variedade de patógenos respiratórios, modulando a resposta inata e adaptativa e mecanismos de regulação inflamatória (Crouch e Wright, 2001). O surfactante pulmonar é um complexo altamente tensoativo que recobre a superfície alveolar do pulmão (Aguilera, 2004). É constituído, de modo geral, por lipídeos e proteínas. Cerca de 80 a 90\% de sua composição em massa é composta por lipídeos, incluindo lipídeos neutros e fosfolipídeos (Rebello et al., 2002; Goerke, 1998). As proteínas representam cerca de 10\% da massa do surfactante, tendo sido reconhecidas quatro proteínas, SP-A, SP-B, SP-C e SP-D 
(LeVine, 1999). As três primeiras são importantes para a estrutura, homeostasia e atividade superficial do surfactante. A SP-D em conjunto com a SP-A tem funções imunomodulatórias (Aguilera, 2004).

As proteínas SP-A e SP-D são consideradas componentes importantes na imunidade natural do pulmão. Existem evidencias de que a SP-A modula a resposta celular induzida por polissacarídeos, mediante interação direta com CD14. Tem-se encontrado que as colectinas pulmonares se ligam a CD14 e alteram a interação com os lipopolissacarídeos e que nesta união participam diretamente os domínios da SP-A e o domínio tipo lecitina da SP-D. É reconhecido o componente peptídico de CD14 pela SP-A e o componente carboidrato de CD14 pela SP-D (Pryhuber, 1998; Sano et al., 2000).

A ausência de SP-A prejudica a eliminação, tanto de bactérias como de vírus dos pulmões, facilitando a disseminação sistêmica de infecções (Rebello et al., 2002; LeVine et al., 1999). A SP-A não está presente nos preparados de surfactante de origem animal, obtidos por processo de extração lipídica, e não tem atividade de redução da tensão superficial (Crouch, 1998). Porém, substâncias como o surfactante, naturais ou sintéticas, atraem células imunes competentes para o local da injeção quando usadas como adjuvante em vacina (Ascarateil e Dupuis, 2006).

O Instituto Butantan desenvolveu tecnologia para produção de surfactante pulmonar de origem suína (Kubrusly et al., 2000), atualmente com a metodologia implantada no setor de produção. Essa preparação apresenta resultados bastante satisfatórios quanto à sua função na tensão alveolar, e vem sendo testada em várias vacinas, para avaliação de sua atividade adjuvante.

\subsubsection{Monofosforil lipídeo A}

Os estudos que inspiraram o desenvolvimento de monofosforil lipídeo A (MPL) como adjuvante começaram há 60 anos, quando foi noticiado que a resposta imune para vacinas de toxóides de difteria e tétano foi melhorada com a adição de uma bactéria gram-negativa (Greenberg e Fleming, 1947). O lipopolissacarídeo, principal componente da parede celular de 
bactérias gram-negativas (Baldridge e Crane, 1999; Bentala et al., 2002), foi isolado e confirmado como o material responsável pelo aumento da resposta imune (Landy et al., 1955; Webster et al., 1955). Desde então muitos estudos têm demonstrado a potencial atividade adjuvante do LPS, ainda que sua toxicidade inerente impossibilite seu uso como um adjuvante de vacina.

O LPS está fixado na superfície externa da membrana externa e é constituído por 3 componentes: o lipídeo A, o núcleo oligossacarídeo e o antígeno-O (Feulner, 2003). A porção lipídeo A do LPS é a responsável pelas suas propriedades imunoestimulatórias e de toxicidade (Baldridge e Crane, 1999; Jiang et al.,2007). Apesar de não se conhecer a atividade biológica da região externa do LPS, acredita-se que possua epitopos que atraem anticorpos (Rietschel et al., 1992).

O monofosforil lipídeo A é um derivado destoxificado da porção lipídeo A do LPS (Figura 2), mantendo suas atividades adjuvantes e imunoestimulatórias. O MPL é diferente do LPS na estrutura, na atividade biológica e habilidade de induzir certas citocinas (Martin et al., 2003). Enquanto o LPS induz a produção das citocinas IL-1, IL-6, IL-8 e fator de necrose tumoral- $\alpha$ (TNF- $\alpha$ ), o MPL induz a produção de IL-10, IL-12 e INF- $\gamma$ (Bentala et al., 2002; Jiang et al., 2007; Martin et al., 2003). O sítio de ação do MPL é o receptor TLR4/MD-2 (toll like receptor 4/ MD-2), que induz citocinas inflamatórias através do NFKB (Fritzgerald e Golenblock, 2007; Mata-Haro et al., 2007; O’Neill, 2006; Persing et al., 2002). Como o TLR4 está presente em células apresentadoras de antígenos, o efeito imunomodulatório do MPL é a estimulação de TLR4 no local de deposição do antígeno (Persing et al., 2002). Dessa forma, o modo de ação deste componente é através da indução da resposta inata dependente de TLR4, e conseqüente indução da resposta imune adaptativa tipo Th1 (Geurtsen et al., 2007; Persing et al., 2002). Segundo Caglar et al. (2005) a imunização de camundongos com toxóide diftérico na presença de MPL (derivado de LPS de Salmonella minnesota Re595) induziu altos níveis de anticorpos contra toxina diftérica, sugerindo que o MPL pode ser um candidato potencial como adjuvante no futuro. 


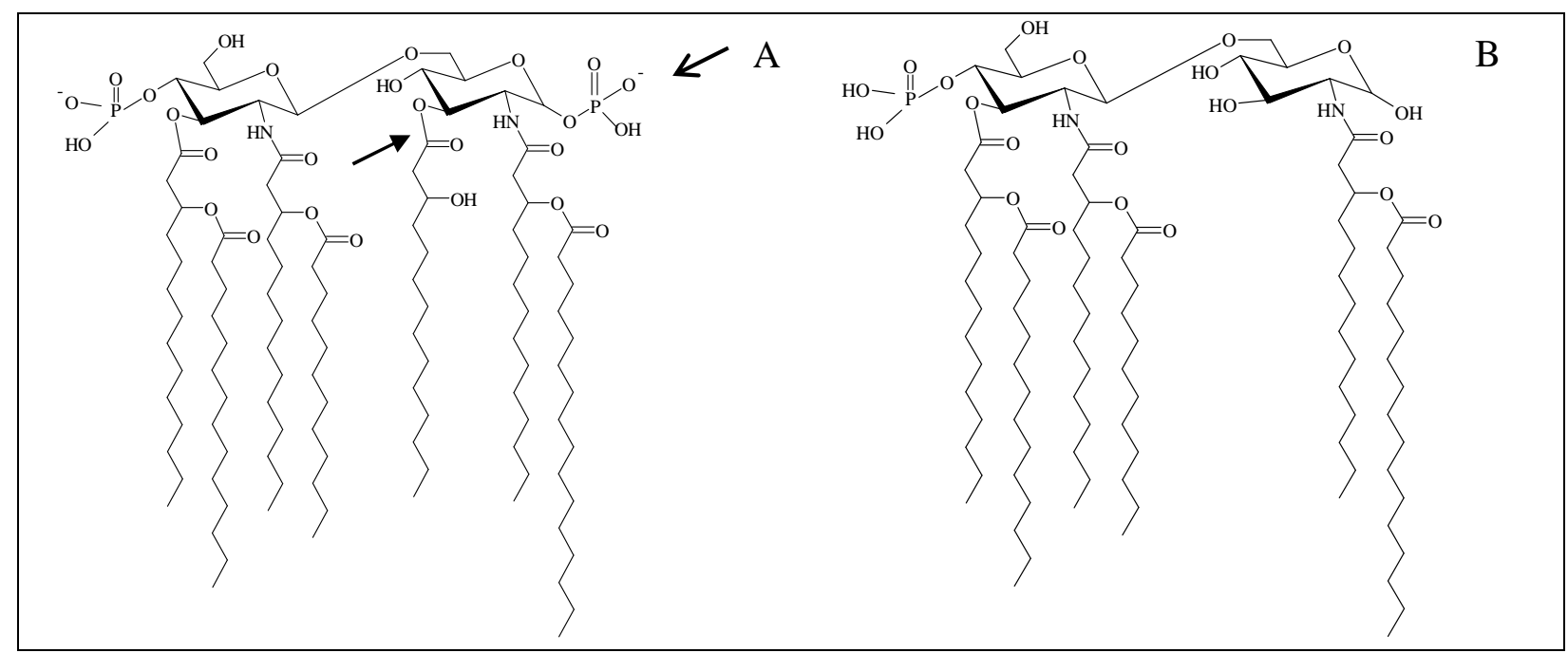

Figura 2 - Estrutura química do lipídeo A (A) e do monofosforil lipídeo A (B), ambos de Salmonella minnesota. A seta aberta indica o grupo fosfato e a seta fechada indica a cadeia lateral, estruturas retiradas após extração orgânica do monofosforil lipídeo A.

FONTE: Figura A modificada de Feulner, 2003 e figura B modificada de Persing et al., 2002.

\subsubsection{BCG}

A vacina BCG foi desenvolvida entre 1906 e 1919, por Camille Calmette e Albert Guérin no Instituto Pasteur (Paris). Esses pesquisadores obtiveram uma cepa atenuada do Mycobacterium bovis original, após 13 anos de passagens sucessivas em meios de cultura, realizadas a cada três semanas, perfazendo o total de 231 passagens. A partir de 1921, a vacina produzida com $M$. bovis atenuado passou a ser utilizada em humanos, recebendo o nome de BCG (Bacilo Calmette-Guérin) (Pereira et al., 2007; Dias et al., 2000). A utilização da vacina BCG foi adotada largamente a partir de 1920, incentivada pela Organização Mundial da Saúde (OMS) e United Nations Children's Fund (UNICEF) a partir de 1948 (Pereira et al., 2007).

No Brasil, o Ministério da Saúde recomenda a aplicação da vacina BCG em maternidades para recém-nascidos sadios com peso superior a $2 \mathrm{~kg}$; crianças HIV-soropositivas; filhos de mães com AIDS, tuberculino-negativas e sem sintomas; profissionais de saúde não reatores; militares não reatores; população indígena. Duas doses são recomendadas para contatos intradomiciliares de hanseníase. As contra-indicações são: recém-nascidos com peso inferior a $2 \mathrm{~kg}$; afecções 
dermatológicas no local da vacina ou generalizadas; imunodeprimidos; adultos HIV soropositivos, independente de sintomas; imunodeficiência congênita (Pereira et al., 2007).

Hoje, o BCG é a vacina mais utilizada no mundo, com pouquíssimos efeitos adversos sérios, o que o torna um bom candidato a veículo para apresentação de antígenos no desenvolvimento de vacinas. O BCG induz a produção de resposta inflamatória, constituída por macrófagos e linfócitos T que secretam citocinas, como INF- $\gamma$, e TNF- $\alpha$ (Dias et al., 2000). Nascimento et al. (2000) observou que células esplênicas de animais imunizados com vacina de BCG expressando a subunidade S1 da toxina pertussis mostraram elevada produção de IFN- $\gamma$, tão bem como aumento da proliferação, após estímulo com PT, caracterizando resposta celular tipo Th1. O BCG é um dos mais efetivos adjuvantes conhecidos para a indução de imunidade celular em animais e humanos; a vacinação intraperitoneal com BCG expressando o fragmento $\mathrm{C}$ da toxina tetânica demonstrou a indução de respostas humoral e celular, inclusive em tecidos mucosos (Mazzantini et al., 2004). 


\section{CONCLUSÕES}

$\mathrm{A}$ variação do componente pertussis ( $\mathrm{Pa}$ e $\mathrm{P}_{\text {low }}$ ) não alterou a resposta induzida pelos toxóides diftérico e tetânico na vacina tríplice bacteriana, tanto em níveis de anticorpos contra os antígenos vacinais, quanto em sua capacidade soroneutralizante. A nosso ver, as novas formulações de vacinas tríplices utilizando o componente pertussis acelular ou o componente pertussis celular, menos reatogênico, $\mathrm{P}_{\text {low, }}$ poderiam substituir a vacina pertussis celular tradicional na vacina tríplice bacteriana com a mesma eficácia. A $\mathrm{P}_{\text {low }}$ está sendo ensaiada em crianças, mas tanto ela quanto a Pa podem ser avaliadas em ensaio de campo, para possível administração como reforço para adolescentes e adultos, já que a vacina pertussis celular tradicional não é recomendada para essas faixas etárias, devido à sua reatogenicidade.

$\mathrm{Na}$ avaliação dos adjuvantes nas diferentes formulações das vacinas tríplices bacterianas, o MPL sugeriu um equilíbrio entre as respostas Th1 e Th2. A substituição do hidróxido de alumínio pelo MPL poderia levar a uma resposta mais próxima à induzida pela infecção nativa e não acarretaria maiores custos para o produto final, já que é um produto obtido a partir do cultivo da Bordetella pertussis, no mesmo processo de produção da vacina tradicional no próprio Instituto Butantan, tornando esta nova formulação economicamente viável. A vitamina A também poderia ser uma alternativa de adjuvante na formulação da vacina tríplice, com ação semelhante à do hidróxido de alumínio, é menos reatogênica e pode atuar como suplemento contra a hipovitaminose A.

A combinação da vacina BCG com os componentes pertussis acelular ou pertussis low, sugeriu, como esperado, uma tendência de resposta celular, principalmente induzida pela formulação da $\mathrm{P}_{\text {low }}$ adicionada de BCG e MPL. A combinação dessas vacinas seria uma estratégia interessante na vacinação de recém nascidos, e deve ser estudada mais detalhadamente. 


\section{REFERÊNCIAS}

Abbas AK, Lichtman AH, Pober JS. Imunologia celular e molecular. 4. ed. Rio de Janeiro: Editora Revinter; 2003.

Aguilera GMH. Genetica molecular del surfactante pulmonary. Revista Habanera de Ciências Médicas. 2004;3(10).

Ascarateil S, Dupuis L. Surfactants in vaccine adjuvants: description and perspectives. Vaccine. 2006;24(2):83-85.

Authier FJ, Sauvat S, Christov C, Chariot P, Raisbeck G, Poron MF, Yiou F, Gherardi R. $\mathrm{Al}(\mathrm{OH})_{3}$-adjuvanted vaccine-induced macrophagic myofasciitis in rats is influenced the genetic background. Neuromuscular Disorders. 2006;16:347-52.

Baldridge JR, Crane RT. Monophosphoryl Lipid A (MPL) formulations for the next generation of vaccines. Methods. 1999;19:103-07.

Baldridge JR, McGowan P, Evans JT, Cluff C, Mossman S, Johnson D, Persing D. Taking a Toll on human disease: Toll-like receptor 4 agonists as vaccine adjuvants and monotherapeutic agents. Expert Opinion on Biological Therapy. 2004;4:1129-38.

Barreto L, Gasparini R, Meekison W, Noya F, Young L, Mills E. Humoral immunity 5 years after booster immunization with an adolescent and adult formulation combined tetanus, diphtheria, and 5-component acellular pertussis vaccine. Vaccine. 2007;25:8172-79.

Baylor NW, Egan W, Richman P. Aluminum salts in vaccines - US perspective. Vaccine. 2002;20:S18-23.

Bentala H, Verweij WR, Van der Vlag AH, Weemaes A M L, Meijer DKF, Poelstra K. Removal of phosphate from lipid A as a strategy to detoxify lipopolysaccharide. Shock. 2002;18(6):56166.

\footnotetext{
* De acordo com:

International Committee of Medical Journal Editors. Uniform requirements for manuscripts submitted to Biomedical Journal: sample references. Available from: http://www.icmje.org [2007 May 22].
} 
Brennan FR, Dougan G. Non-clinical safety evaluation of novel vaccines and adjuvants: new products, new strategies. Vaccine. 2005;23:3210-22.

Caglar K, Aybay C, Ataoglu H. Effect of monophosphoryl lipid A on antibody response to diphtheria toxin and its subunits. APMIS. 2005;113:256-63.

Campins-Martí M, Cheng HK, Forsyth K, Guiso N, Halperin S, Huang LM, Mertsola J, Oselka G, Ward J, Wirsing von König CH, Zepp F. Recommendations are needed for adolescent and adult pertussis immunization: rationale and strategies for consideration. Vaccine. 2002;20:64146.

Carvalho AP, Pereira EMC. Acellular pertussis vaccine for adolescents. Jornal de Pediatria. 2006;83(3):S15-23.

CDC. Preventing tetanus, diphtheria, and pertussis among adults: use of tetanus toxoid, reduced diphtheria toxoid and acellular pertussis vaccine (Tdap): recommendations of the Advisory Committee on Immunization Practices (ACIP) and Recommendations of ACIP supported by the Healthcare Infection Control Practices Advisory Committee (HICPAC), for use of Tdap among health-care personnel. MMWr 2006; 55(No.RR17). Disponível em: http://www.cdc.gov/mmwr/preview/mmwrhtml/rr5517a1.htm [2008 Nov 19].

Cherry JD, Olin P. The science and fiction of pertussis vaccines. Pediatrics. 1999;104:1381-83.

Cherry JD. Epidemiological, clinical, and laboratory aspects of pertussis in adults. Clinical and Infection Disease. 1999;28(2):S112-17.

Cherry JD. The Science and Fiction of the "Resurgence" of Pertussis. Pediatrics. 2003;112;40506.

Clements CJ, Griffths E. The global impact of vaccines containing aluminium adjuvants. Vaccine. 2002;20: S24-33.

Cox JC, Coulter AR. Adjuvants - a classification and review of their modes of action. Vaccine. 1997;15(3):248-56. 
Crouch E, Wright JR. Surfactant proteins A and D and pulmonary host defense. Annual Review Physiology. 2001;63:521-54.

Crouch EC. Structure, biologic properties and expression of surfactant protein D (SP-D). Biochimica at Biophysica Acta. 1998;1408:278-89.

De Becker G, Moulin V, Pajak B, Bruck C, Francotte M, Thiriart C, Urbain J, Moser M. The adjuvant monophosphoryl lipid $\mathrm{A}$ increases the function of antigen-presenting cells. International Immunology. 2000;12:807-15.

Denöel P, Godfroid F, Guiso N, Hallander H, Poolman J. Comparison of pertussis vaccinesinduced immunity against infection due to Bordetella pertussis variant isolates in a mouse model. Vaccine. 2005;23:46-47.

Dias WO, Esteves MI, Horton DSPQ, Gebara VCBC, Abreu PAE, Risoleo L, Silva APG, Raw I. Adjuvant activity of acellular pertussis vaccine in tetanus vaccine. Immunology. 2004a; 333-36.

Dias WO, Horton DSPQ, Furuyama N, Quintilio W, Sakauchi MA, Raw I; Fundação Butantan. Processo de Obtenção de Vacina Pertussis Acelular. Brasil patente BR INPI PI 0202458-6. 2002a.

Dias WO, Horton DSPQ, Gebara VCBC, Furuyama N, Risoléo L, Ferreira VRF, Raw I. Vitamin $A$ as adjuvant to the mouse immune response to pertussis, tetanus and diphtheria vaccines. Biotechnology Letters. 2002b;24:1515-18.

Dias WO, Horton DSPQ, Takahashi CM, Raw I. A Bordetella pertussis acellular vaccine candidate: antigenic characterization and antibodies induction. Brazilian Journal of Medical and Biological Research. 1994;27:2607-11.

Dias WO, Kubrusly FS, Esteves MI, Horton DSPQ, Furuyama N, Quintilio W, Sakauchi MA, Leme E, Prado SMA, Mendes E, Higashi H, Raw I; Fundação Butantan - Processo de Obtenção de Nova Vacina Pertussis Celular. Brasil patente BR INPI PI 0402630-6. 2004b.

Dias WO, Leite LCC, Horton DSPQ, Sakauchi MA, Kubrusly FS, Furuyama N, Nascimento IP, Quintilio W, Higashi HG, Raw I. New approaches in pertussis vaccines for developing countries In: Communicating Current Research and Educational Topics and Trends in Applied Microbiology. 2007;3:668-72. 
Dias WO, Mazzantini RP, Miyaji EM, Nascimento IP, Horton DSQ, Leite LCC. Vacinas de BCG recombinante-DTP. Revista Biotecnologia Ciência e Desenvolvimento. 2000;7:24-28.

Feulner JA. Identification of acyloxyacyl hydrolase, A lipopolysaccharide-detoxifying enzyme, in the murine urinary tract [Ph. D. Thesis]. Dallas: The University of Texas Southwestern Medical Center at Dallas; 2003.

Fritzgerald KA, Golenblock DT. Immunology. The shape of things to come. Science. 2007;316 (5831):1628-32.

Furuyama, N. Avaliação de procedimento de destoxificação de toxina pertussis, em vacina pertussis acelular desenvolvida no Instituto Butantan [dissertação (Mestrado em Biotecnologia)]. São Paulo: Programa de Pós-Graduação Interunidades em Biotecnologia USP/Instituto Butantan/ IPT; 2001.

Geurtsen J, Banus HA, Gremmer ER, Ferguson H, de la Fonteyne-Blankestijn LJJ, Vermeulen JP, Dormans JAMA, Tommassen J, van der Ley P, Mooi FR, Vandebriel RJ. Lipopolysaccharide analogs improve efficacy of acellular pertussis vaccine and reduce type I hypersensitivity in mice. Clinical and Vaccine Immunology. 2007;14(7):821-29.

Geurtsen J, Fransen F, Vandebriel RJ, Gremmer ER, la Fonteyne-Blankestijn LJJ, Kuipers B, Tomasen J, van der Ley P. Supplementation of whole-cell pertussis bacines with lipopolysaccharide analogs: modification of vaccine-induced immune responses. Vaccine. 2008;26:899-906.

Gherardi RK, Coquet M, Cherin P, Belec L, Moretto P, Dreyfus PA, Pellissier JF, Chariot P, Authier FJ. Macrofagic myofasciitis lesions assess long-term persistence of vaccine-derived aluminium hydroxide in muscle. Brain. 2001;124:1821-31.

Glenny AT, Pope CG, Waddington H, Wallace U. The antigenic value of toxoid precipitated by potassium alum. The Journal of Pathology and Bacteriology. 1926;29:38-39.

Goerke J. Pulmonary surfactant: functions and molecular composition. Biochimica at Biophysica Acta. 1998;1408:79-89.

Gomariz RP, Arranz A, Juarranz Y, Gutierrez-Cañas I, Gomez MG, Leceta J, Martinez C. Regulation of TLR expression, a new perspective for the role of VIP in immunity. Peptides. 2007;28:1825-32. 
Goto N, Kato H, Maeyama J, Eto K, Yoshihara S. Studies on the toxicities of aluminium hydroxide and calcium phosphate as immunological adjuvants for vaccines. Vaccine. 1993;11(9):914-18.

Greco D, Salmaso S, Mastrantonio P. A controlled trial of two acellular vaccines and one wholecell vaccine against pertussis. The New England Journal of Medicine. 1996;334:341-48.

Greenberg L, Fleming, DS. Increased efficiency of diphtheria toxoid when combined with pertussis vaccine.Can. Journal Public Health. 1947;38:279-82.

Gustafsson L, Hallander HO, Olin P, Reizenstein E, Storsaeter J. A controlled trial of a twocomponent acellular, a five-component acellular, and a whole-cell pertussis vaccine. The New England Journal of Medicine. 1996;334:349-55.

HogenEsch H. Mechanisms of stimulation of the immune response by aluminum adjuvants. Vaccine. 2002;20:S34-39.

Hussey GD, Klein M. A randomized, controlled trial of vitamin A in children with severe measles. New England Journal of Medicine. 1990;323:160-64.

Ismaili J, Rennesson J, Aksoy E, Vekemans J, Vincart B, Amraoui Z, Van LF, Goldman M, Dubois PM. Monophosphoryl lipid A activates both human dendritic cells. The Journal of Immunology. 2002;168:926-32.

Jason J, Archibald LK, Nwanyanwu OC, Sowell AL, Buchanan I, Larned J, Bell M, Kazembe PN, Dobbie H, Jarvis WR. Vitamin A levels and immunity in humans. Clinical and Diagnostic Laboratory Immunology. 2002;9(3):616-21.

Jefferson T, Rudin M, Di Pietrantonj C. Adverse events after immunization with aluminiumcontaining DTP vaccines: systematic review of the evidence. The Lancet Infectious Diseases. 2004;4:84-90.

Jiang ZH, Budzynski WA, Qiu D, Yalamati D, Koganty RR. Monophosphoryl lipid A analogues with varying 3-o substitution: synthesis and potent adjuvant activity. Carbohydrate Research. 2007;342:784-96. 
Kubrusly FS, Dias WO, Esteves MI, Furuyama N, Horton DSPQ, Quintilio W, Sakauchi MA, Prado MAS, Mendes E, Higashi HG, Raw I. Process for obtention of new cellular pertussis vaccine. WO/2006/002502. 2006.

Kubrusly FS, Netto SL, Iourtov D, Raw I, Araújo PS. A natural surfactant from pig lungs. Biotechnology Letters. 2000;22:1251-53.

Landy M, Johnson, AG, Webster ME, Sagin JF. Studies on the O Antigen of Salmonella typhosa II. Journal of Immunology. 1955;74:466-78.

Latif R, de Rosbo NK, Amarant T, Rappuoli R, Sappler G, Ben-Nun A. Reversal of the $\mathrm{CD} 4_{1} / \mathrm{CD} 8_{1} \mathrm{~T}$-cell ratio in lymph node cells upon in vitro mitogênico stimulation by highly purified, water-soble S3-S4 dimer of pertussis toxin. Infection and Immunity. 2001;69(5):307381.

Leef M, Elkins KL, Barbic J, Shahin RD. Protective immunity to Bordetella pertussis requires both B cells and CD4+ T cells for key functions other than specific antibody production. The Journal of Experimental Medicine. 2000;191(11):1841-52.

Leung AK, Robson WL, Davies HD. Pertussis in adolescents. Advances in therapy. 2007;24(2):353-61.

LeVine AM, Kurak KE, Wright JR, Watford WT, Bruno MD, Ross GF, et al. Surfactant protein A (SP-A) binds group B streptococcus enhancing phagocytosis and clearance from lungs of surfactant protein A deficient mice. American Journal Respiratory Cell and Molecular Biology. 1999;20(2):279-86.

Lima MGS. Uso de óleos vegetais como adjuvantes da resposta imunológica em procedimentos de imunização/vacinação. Departamento de bioquímica e biologia molecular. Disponível em: http://64.233.169.104/search?q=cache:jqM2aWE1K04J:sbbq.iq.usp.br/arquivos/regional/2002/cd resumo/Palestras/028.pdf+sbbq.iq.usp.br/arquivos/regional/2002/cdresumo/palestras/028.pdf\&hl $=\mathrm{pt}-\mathrm{BR} \& \mathrm{ct}=\mathrm{clnk} \& \mathrm{~cd}=1 \& \mathrm{gl}=\mathrm{br}[2008 \mathrm{jan} 08]$.

Lin R, Jones TC. Vaccine adjuvants: A new hope for effeticve immunization. Brazil Journal Infection Disease. 1997;1(3):106-22. 
Ma Y, Ross AC. The anti-tetanus immune response of neonatal mice is augmented by retinóico acid combined with polyriboinosinic: polyribocytidylic acid. Proceedings of the National Academy of Science of United States of America. 2005;102(38):13556-61.

Martin M, Michalek SM, Katz J. Role of innate immune factors in the adjuvant activity of monophosphoryl lipid A. Infection and Immunity. 2003 May;71(5):2498-507.

Marzzoco A, Torres BB. Bioquímica Básica. 2.ed. Rio de Janeiro: Editora Guanabara Koogan; 1999.

Mata-Haro V, Caglar C, Martin M, Chilton PM, Casella CR, Mitchelli TC. The vaccine adjuvant monophosphoryl lipid A as a TRIF-Biased agonist of TLR4. Science. 2007;316:1628-32.

Matos DCS, Narcovistz R, Cabello PH, Georgini RA, Sakauchi D, Silva LL. Immunogenicity test of tetanus component in adsorbed vaccines by toxin binding inhibition test. Mem Instituto Oswaldo Cruz. 2002;97(6):909-13.

Maxwell JR, Ruby C, Kerkvliet NI, Vella AT. Contrasting the role of costimulation and the natural adjuvant lipopolysaccharide during the induction of $\mathrm{T}$ cell immunity. The Journal of Immunology. 2002;168:4372-81.

Mazzantini RP, Miyaji EM, Dias WO, Sakauchi D, Nascimento ALTO, Raw I, Winter N, Gicquel B, Rappouli Leite, LCC. Adjuvant activity of Mycobacterium bovis BCG expressing $\mathrm{CRM}_{197}$ on the immune response induced by BCG expressing tetanus toxin fragment $\mathrm{C}$. Vaccine. 2004;22:740-46.

Mielcarek N, Debrie AS, Raze D, Bertout J, Rouanet C, Younes AB, Creusy C, Engle J, Goldman WE, Locht C. Live attenuated B. pertussis as a single-dose nasal vaccine against whooping cough. PloS Pathogens. 2006;2(7):662-70.

Mills KHG. Immunity to Bordetella pertussis. Microbes and Infection. 2001;3:655-77.

Mitchell TC, Hildeman D, Kedl RM, Teague TK, Schaefer BC, White J, Zhu Y, Kappler J, Marrack P. Immunological adjuvants promote activated T cell survival via induction of Bcl-3. Nature Immunology. 2001;2:397-402. 
Mizuno D, Kurihara MI, Ichinomiya T, Kubo I, Kido H. Modified pulmonary surfactant is a potent adjuvant that stimulates the mucosal $\operatorname{IgA}$ production in response to the influenza virus antigen. The Journal of Immunology. 2006;1122-30.

Nascimento I, Dias WO, Quintilio W, Christ A, Moraes J, Vanceto M, Santos GR, Raw I, Leite L. Neonatal immunization with a single dose of recombinant BCG expressing subunit S1 from pertussis toxin induces complete protection against Bordetella pertussis intracerebral challenge. Microbes and Infection. 2008;10:198-202.

Nascimento IP, Dias WO, Mazzantini RP, Miyaji EN, Gamberini M, Quintilio W, Gebara VC, Cardoso DF, HO PL, Raw I, Winter N, Gicquel B, Rappuoli R, Leite LCC. Recombinant Mycobacterium bovis BCG expressing pertussis toxin subunit $\mathrm{S} 1$ induces protection against an intracerebral challenge with live Bordetella pertussis in mice. Infection and Immunity. 2000;68(9):4877-83.

Naylor PH, Hadden JW. T cell targeted immune enhancement yields effective T cell adjuvants. International immunopharmacology. 2003;3:1205-15.

Ntezayabo B, De Serres G, Duval B. Pertussis resurgence in Canada largely caused by a cohort effect. Pediatric Infection Disease Journal. 2003;22:22-7.

O'Neill LAJ. How Toll-like receptors signal: what we know and what we don't know. Current Opinion in Immunology. 2006;18:3-9.

Pachichero ME, Casey JR. Acellular pertussis vaccines for adolescents. Pediatric Infectious Disease Journal. 2005;24(6 suppl):S117-26.

Pashine A, Valiante NM, Ulmer JB. Targeting the innate immune response with improved vaccine adjuvants. Nature Medicine. 2005;11(4):S63-68.

Pereira SM, Dantas OMS, Ximenes R, Barreto ML. Vacina BCG contra tuberculose: efeito protetor e políticas de vacinação. Revista de Saúde Pública. 2007;41 supl 1.

Persing DH, Coler RN, Lacy MJ, Johnson A, Baldridge JR, Hershberg RM, Reed SG. Taking toll: lipid A mimetics as adjuvants and immunomodulators. Trends in Microbiology. 2002;10(10):S32-37. 
Programa nacional de suplementação de vitamina A. Disponível em: http://nutricao.saude.gov.br/vita.php [2005 out 20].

Pryhuber GS. Regulation and function of pulmonary surfactant protein B. Molecular Genetics and Metabolism. 1998;64:217-28.

Raguckas SE, VandenBussche HL, Jacobs C, Klepser ME. Pertussis resurgence: diagnosis, treatment, prevention, and beyond. Pharmacotherapy. 2007;27(1):41-52.

Raw I, Kubrusly FS, Iourtov D, Sakauchi MA, Santos FL, Dias SC, Darini E, Furuyama N, Prado SMA, Higashi HG. Method to obtain monophosphoryl lipid A from Bordetella pertussis a by-product of the cellular pertussis vaccine production; 2006. (PCTBR2007/000073).

Rebello CM, Proença SM, Troster EJ, Jobe AH. Terapia com surfactante pulmonar exógeno - o que é estabelecido e o que necessitamos determinar. Jornal de Pediatria. 2002;78(2):S215-26.

Recommendations for whole-cell pertussis vaccine. Technical Report Series No 941. World Health Organization WHO, 2007, Annex 6. Disponível em: http://www.who.int/biologicals/publications/trs/areas/vaccines/whole_cell_pertussis/Annex\%206 \%20whole\%20cell\%20pertussis.pdf [2008 nov 04].

Rennels MB, Deloria MA, Pichichero ME, Englund JA, Anderson EL, Steinhoff MC, Decker MD, Edwards KM. Lack of consistent relationship between quantity of aluminum in diphtheriatetanus-acellular pertussis vaccine and rates of extensive swelling reactions. Vaccine. 2002;20:S44-47.

Resende FCB, Passold J, Ferreira SIAC, Zanetti CR, Lima HC. Adjuvantes de vacinas: possibilidades de uso em seres humanos ou animais. Revista Brasileira de Alergia e Imunopatologia. 2004;27(3):116-24.

Rietschel ET, Brade L, Lindner B, and Zahringer U. Molecular biochemistry of lipopolysaccharides. Bacterial Endotoxic Lipopolysaccharides. 1992;3-42.

Ross AC. Vitamin A deficiency and retinoid repletion regulate the antibody response to bacterial antigens and the maintenance of natural killer cells. Clinical Immunology and Immunopathology. 1996;80:S63-72. 
Ross D. Vitamin A plus measles vaccination: the downside of convenience? Lancet. 1995;345:1330-32.

Sano H, Chiba H, Iwaki D, Sohma H, Voelker DR Kuroki Y. Surfactant Proteins A and D bind CD14 by different mechanisms. Journal of Biolical Chemistry. 2000;275(29):22442-51.

Semba RD, Muhilal, Scott AL, Natadisastra G, West Jr KP, Sommer A. Effect of vitamina A supplementation on immunoglobulin $\mathrm{G}$ subclass responses to tetanus in children. Clinical and Diagnostic Laboratory Immunology. 1994;1(2):172-5.

Sommer A, Davidson FR. Assessment and control of vitamin A deficiency: the Annecy Accords. Journal of Nutritio. 2002;132:2845-50.

Sonobe MH, Trezena AG, Guilhen FB, Takano VL, Fratelli F, Sakauchi D, Moraes JF, Prado, SMA Higashi HG. Determination of low tetanus or diphtheria antitoxin titers in sera by a toxin neutralization assay and a modified toxin-binding inhibition test. Brazilian Journal of Medical and Biological Research. 2007;40: 69-76.

Teste de Mann Whitney. Disponível em: http://elegans.swmed.edu/ leon/stats/utest.html [2006 Abr 14].

Thompson HSG, Davies ML, Watss MJ, Mann AE, Holding FP, O'Neill TO, Beech JT, Thompson SJ, Leesman GD, Ulrich JT. Enhanced immunogenicity of a recombinant genital warts vaccine adjuvanted with monophosphoryl lipid A. Vaccine. 1998;16(20):1993-9.

Vacinas Inativadas: os adjuvantes. Disponível em: http://www.fortdodge.com.br/fd/aves/ manuais/aves01/folheto_ina/pagina2.htm [2008 jan 08].

Vacinas: Vacina Tríplice Bacteriana - Vacina combinada contra a difteria, a coqueluche e o tétano. Disponível em: http://www.vacinas.org.br/vacinas20.htm [2008 jun 02].

Vella AT, McCormack JE, Linsley PS, Kappler JW, Marrack P. Lipopolysaccharide interferes with the induction of peripheral T cell death. Immunity. 1995;2:261-70.

Vitamin A and your bones. Harvard Men's Health Watch (July, 2005). Disponível em: http://www.periodicos.capes.gov.br/portugues/index.jsp [2005 Set 26]. 
Vitamina A. Disponível em: http://www.emedix.com.br/vit/vit002 1f vitaminaa.php [2008 jan 13].

von König CH, Halperin S, Riffelmann M, Guiso N. Pertussis of adults and infants. Lancet. Infection Disease. 2002;2(12):744-50.

Webster ME, Sagin JF, Landy M, Johnson AG. Studies on the O antigen of Salmonella typhosa. I. Purification of the antigen. Journal of Immunology. 1955;74:455-65.

Zorzeto TQ, Ramalho VD, Lima SCB, Silva MTN, Mazzola TN, Carniel EF. Gamma delta ${ }^{+}$T cell expansion in infants vaccinated with low lipopolysacharide cellular pertussis (DTwPlow) or whole cell pertussis (DTwP). In: Vaccine Congress; Amsterdam: Netherlands; 2007a. Abstract Book poster... Amsterdam: Netherlands; 2007a. p.163.

Zorzeto TQ, Rollo AF, Dias WO, Stephano MA, Carniel EF, Silva MTN. Immunogenicity of a low lipopoysacharide (LPS) cellular Bordetella pertussis vaccine among infants. In: Vaccine Congress; Amsterdam: Netherlands; 2007a. Abstract Book poster... Amsterdam: Netherlands; 2007b. p.164. 